doi: $10.15407 /$ ujpe62.01.0051

S. GURUPARAN,${ }^{1}$ V. RAVICHANDRAN,${ }^{2}$ V. CHINNATHAMBI, ${ }^{2}$ S. RAJASEKAR ${ }^{3}$

${ }^{1}$ Department of Chemistry, Sri K.G.S. Arts College (Srivaikuntam 628 619, Tamilnadu, India)

2 Department of Physics, Sri K.G.S. Arts College

(Srivaikuntam 628 619, Tamilnadu, India)

${ }^{3}$ School of Physics, Bharathidasan University

(Tiruchirapalli 620 024, Tamilnadu, India)

\title{
COEXISTENCE OF MULTIPLE ATTRACTORS, HYSTERESIS, AND VIBRATIONAL RESONANCE IN THE CLASSICAL MORSE OSCILLATOR DRIVEN BY AN AMPLITUDE MODULATED SIGNAL
}

PACS 05.45.-a, 05.90.+m, 46.40.Ff.

\begin{abstract}
We consider the classical Morse oscillator driven by an amplitude modulated signal with two widely different frequencies $\omega$ and $\Omega$, where $\Omega \gg \omega$. The dynamics of such oscillator is numerically studied for a specific set of parameters. We show the occurrence of coexistence of several period-T orbits, bifurcations of them, and hysteresis and vibrational resonance phenomena. We characterize the periodic and chaotic orbits, hysteresis, and vibrational resonance with the use of the bifurcation diagram and response amplitude.
\end{abstract}

Keywords: classical Morse oscillator, coexistence of multiple attractors, hysteresis, vibrational resonance, amplitude modulated signal.

\section{Introduction}

In this paper, we consider the damped classical Morse oscillator. Under the excitation by an AM signal, it is described by the equation

$\ddot{x}+d \dot{x}+\frac{d V(x)}{d x}=(f+2 g \cos \Omega t) \sin \omega t$,

where

$V(x)=\frac{1}{2 \alpha} \beta e^{-\alpha x}\left(e^{-\alpha x}-2\right)$

is the Morse potential $[1,2]$. Here, $\beta$ is the dissociation energy, $\alpha$ is the range parameter, $d$ is the damping coefficient, $f$ is the unmodulated carrier amplitude, $2 g$ is the degree of modulation, $\Omega$ and $\omega$ are the two frequencies of the force with $\Omega \gg \omega$. Figure 1

(C) S. GURUPARAN, V. RAVICHANDRAN, V. CHINNATHAMBI, S. RAJASEKAR, 2017

ISSN 2071-0194. Ukr. J. Phys. 2017. Vol. 62, No. 1 depicts the form of the Morse potential for three different values of $\beta$, namely $\beta=2.0,1.0,0.5$, and for $\alpha=1$. The potential $V(x)$ has one local minimum at $x=0$ and $V(x) \rightarrow \infty$ as $x \rightarrow-\infty$, while it becomes zero as $x \rightarrow \infty$. The Morse potential is widely used to provide an approximate potential energy function for diatomic molecules and to describe the potential energy surface along a bond stretching direction in polyatomic molecules $[1,2]$.

The Morse oscillator is the most prominent example of an anharmonic oscillator that has found wide applications. The driven Morse oscillator without damping $(d=0)$ is characterized by an equation frequently used in theoretical chemistry to describe the photodissociation of molecules $[3,4]$. The damped and driven Morse oscillator (Eq. (1)) can serve as a rough model for the interatomic potential and fitting the vibrational spectra of diatomic molecules. It is also used to describe the infrared multiphoton exci- 


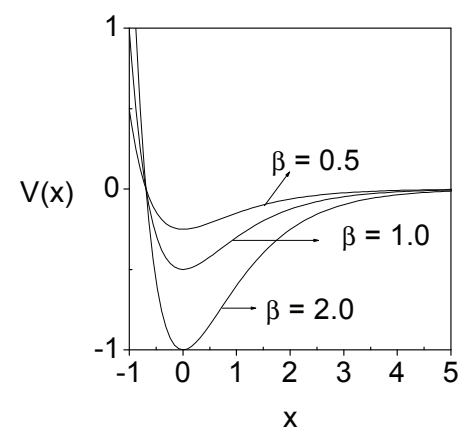

Fig. 1. Morse potential curve for three values of $\beta$ with $\alpha=1$

tation, laser isotopic separation, anomalous gains observed in the stimulated Raman emission, and the dissociation of van der Waals complexes [3-8]. In recent years, many studies were focused within the classical, semiclassical, and quantum mechanical methods on the Morse oscillator dynamics. In particular, Abirami et al. [9] investigated recently the occurrence of a vibrational resonance in both classical and quantummechanical Morse oscillators driven by a biharmonic force. Behnia et al. [10] studied the control over chaos via a slave-master feedback in the classical Morse oscillator. The bifurcations of periodic orbits and its existence are studied both analytically and numerically by Jing et al. [11].

A large class of nonlinear dynamical systems is characterized by the coexistence of multiple attractors in some regions of the parameter space. They lead to the unpredictable behavior of trajectories when a set of parameters is slowly varied through the bifurcation point and are also considered as a source of unpredictability in nonlinear dynamical systems [12-14]. The coexistence of several attractors gives rise to the possibility of hysteresis, that is, the possibility of jumping through the coexisting attractors in a way that is not reversible, when we fix a parameter back to its original value. It is present in the mechanical system, electromagnetism, chemical kinetics, astrochemical cloud models, and nonlinear optics. In particular, the coexistence of attractors and hysteresis have been observed in the generalized Ueda oscillator [15], two coupled overdamped anharmonic oscillators [16], modified Chua's circuit model [17], and the experimental study of Colpitt's oscillator [18].

A weakly nonlinear oscillator exhibiting a periodic response to an external periodic force of frequency $\omega=k \omega_{0}$ (where $\omega_{0}$ is the natural frequency of the oscillator in the absence of an applied force) is said to be in the resonance of order $k$ with the external force, when $\omega \approx k \omega_{0}$. Then the system is close to a resonance of order $k$. The cases $\omega=\omega_{0}$ and $\omega=k \omega_{0}$, $k>1$, are referred as the primary and secondary resonances, respectively. An integer value of $k$ corresponds to the subharmonic resonance. If $k=1 / n$, where $n$ is an integer, then it refers to the superharmonic resonance. Recently, Lukomsky et al. [19] proposed a consecutive scheme for studying the harmonic and subharmonic driven oscillations described by second-order differential equations with arbitrary polynomial nonlinearity.

The study of nonlinear systems subjected to an external periodic force and noise led to several fascinating phenomena. Stochastic resonance [20] is one of such phenomena in which an enhancement of the signal amplitude is observed at an optimum noise intensity. It has been shown both theoretically and experimentally that the amplification of a resonance can be achieved when noise is replaced by a high-frequency periodic force, and the associated effect is called the vibrational resonance $(V R)[9,16,17]$. The analysis of vibrational resonances has attracted a considerable interest in recent years because of its wide variety of applications. The phenomenon of VR was initially presented in ref. [21] and later reported in monostable [22], multistable [23], excitable [24], and spatially periodic potentials [25], maps [26], electronic circuits [17], and time-delayed systems [27]. Recent studies show that VR can also occur at the high-order frequencies. Specifically, if the low frequency of one excitation is $\omega$, the vibrational resonance induced by the high-frequency excitation may occur at frequencies which are multiple of the frequency $\omega$. In a very recent paper, Yang et al. [28] investigated the vibrational subharmonic and superharmonic resonances in an overdamped bistable system driven by two harmonic excitations. In our present work, we will numerically analyze the occurrence of VR due to a highfrequency periodic force with $\Omega \gg \omega$, the coexistence of multiple attractors, and hysteresis in a classical Morse oscillator driven by an amplitude modulated (AM) signal.

The structure of this paper is as follows. In Section 2, we present the damped and driven classical Morse oscillator model and its parameters. We numerically analyze the dynamical behavior of the sys- 
tem driven by an AM signal in Section 3. We show the occurrence of coexistence of multiple attractors and the bifurcations. We also illustrate the presence of the phenomena of hysteresis and vibrational resonance. The conclusions are drawn in Section 4 .

\section{Damped and Driven Classical Morse Oscillator and Parameters Values}

The differential equation which describes the classical Morse oscillator system driven by an AM signal is as follows:

$\ddot{x}+d \dot{x}+\beta e^{-\alpha x}\left(1-e^{-\alpha x}\right)=(f+2 g \cos \Omega t) \sin \omega t$.

The dynamics of a nonlinear system is generally sensitive to the parameters of the system. Since the exact analytic solution of the nonlinear system is not known, we wish to carry out the numerical simulation for certain choices of the parameters. For simplicity, the values of $\Omega$ is chosen as the integral multiples of $\omega$. Different values of $\Omega$ will give the different dynamics. For our numerical study, we fix the values of the parameters at $\alpha=1, d=0.5, \beta=2.0$, 1.5, $0.5, \omega=1$ and $\Omega=10$. The initial values in the numerical calculations are fixed at $x(0)=$ $=0.1$ and $\dot{x}(0)=0$. Equation (3) is solved by the fourth-order Runge-Kutta method with time step size $\Delta t=(2 \pi / \omega) / 200$. Numerical solutions corresponding to 500 first drive cycles are left as transient. We analyzed the behavior of system Eq. (3) by varying the amplitude $f$ of the low-frequency signal and the amplitude $g$ and the frequency $\Omega$ of the highfrequency signal. The numerical results are demonstrated through a bifurcation diagram and the calculation of a response amplitude $Q$. Recently, Ravichandran et al. [29] studied the effect of an AM signal on horseshoe chaos and routes to asymptotic chaos in the Duffing oscillator. Yang et al. [30] investigated the control over VR in a delayed multistable system driven by an AM signal, and Gandhimathi et al. [31] investigated the vibrational and stochastic resonances in two coupled overdamped anharmonic oscillators driven by an AM signal.

\section{Dynamical Behaviors of the System}

In this section, we numerically analyze the dynamical behaviors such as the coexistence of multiple attractors and the phenomena of hysteresis and vibrational
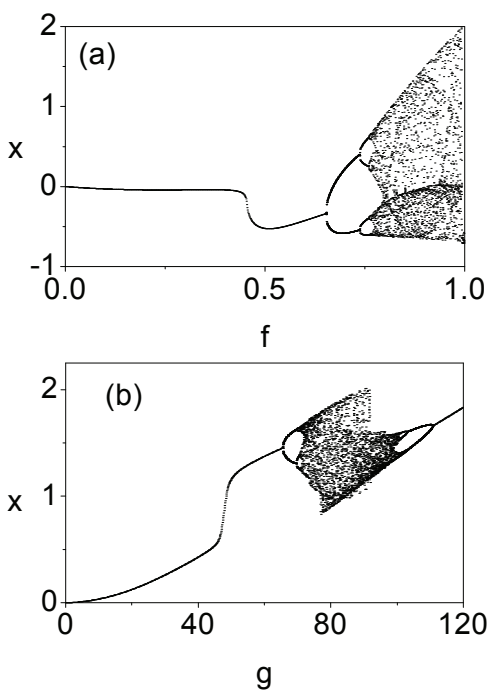

Fig. 2. Bifurcation diagrams for the system described by Eq. (3) and driven by an AM signal when $(a) g=0$ and $(b)$ $f=0$. The other parameters are $d=0.5, \alpha=1.0, \beta=2.0$, $\omega=1.0$ and $\Omega=10$

resonance in the classical Morse oscillator system described by Eq. (3) by varying the amplitudes and frequencies of the AM signal.

\subsection{Coexistence of multiple attractors}

For a range of $f$ and $g$, the system described by Eq. (3) has the coexistence of several attractors. When the control parameter $f$ or $g$ is varied, the system undergoes period-doubling bifurcations leading to chaotic motions at some critical values. The bifurcation diagram for the parameter $f \in[0,1.0]$ with $g=0$ (i.e., the system is driven by the sinusoidal force $f \sin \omega t$ ) is shown in Fig. 2, a. In this bifurcation diagram, the ordinate represents the values of $x(t)$ collected at time $t$ equal to every integral multiple of $2 \pi / \omega$ (Poincaré points) after leaving the sufficient transient evolution. In other bifurcation diagrams with $g \neq 0$ (Fig. 2, b, Fig. 4, and Fig. 5), the period of the force is set to $T=2 \pi /(\Omega+\omega)$. At the critical value of a control parameter $f$, the stability of equilibrium points is exchanged or transformed. This type of bifurcation is called the transcritical bifurcation. At $f=f_{c}=0.44656$, a transcritical bifurcation occurs, at which maximal Lyapunov exponent $\left(\lambda_{m}\right) \approx 0$. When $f$ is further increased from $f=0.44656$, a period-2T limit cycle is developed at $f=0.65291$. The period-2T orbit becomes unsta- 

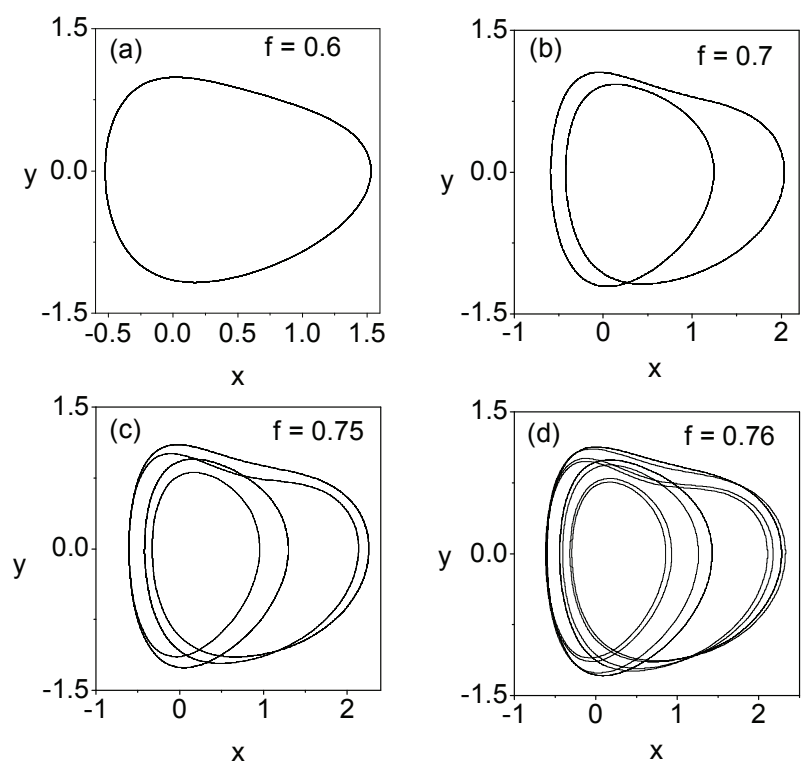

Fig. 3. Period-doubling bifurcation of the Morse oscillator (Eq. 3) driven by an AM signal, when $g=0$. The other parameters are $d=0.5, \alpha=1.0, \beta=2.0, \omega=1.0$, and $\Omega=10$

ble at $f=0.73586$ and gives birth to a limit cycle with period $-2^{2} T$. The bifurcations to a period $-2^{3} T$, period- $-2^{4} T$, period- $2^{5} T$, period $-2^{6} T$, and period $-2^{7} T$ orbits are also observed at $f=0.75873, f=0.76452$, $f=0.76588, f=0.76617$, and $f=0.76623$. This sequence of period-doubling bifurcations accumulates at $f=0.77637$. For $0.77637<f<1.0$, a chaotic motion is found. This shows that the periodic motion and chaotic motion coexist for the range $f \in[0,1]$. Figure 3 shows the phase trajectory of periods $T$, $2 T, 4 T$, and $8 T$ limit cycles from Eq. (3). The approach to chaos via the period-doubling is in agreement with the Feigenbaum constant $(\delta)$ scenario $[32$, 33 which draws on the behavior of nonconservative systems, where the consecutive control intervals tend to a fixed ratio $\delta=4.66992 \ldots$ as an accumulation point is reached. From Fig. 2, $a$, we find the ratios $\delta_{1}=3.62702, \delta_{2}=3.94991, \delta_{3}=4.25735$, $\delta_{4}=4.65432$, and $\delta_{5}=4.66431$ that are in fair agreement with the Feigenbaum constant $(\delta)$, when $n \rightarrow \infty$.

Now, we consider the effect of an AM signal in the Morse oscillator system, by fixing the value of $f=0$ and thereby varying $g$. The obtained bifurcation diagram is shown in Fig. 2, b. We can clearly see the occurrence of the finite period-doubling, reverse perioddoubling, and chaos in Fig. 2, b. At $g=g_{c}=49.2593$, a transcritical bifurcation occurs. When $g$ is increased from $g_{c}=49.2593$, this orbit becomes unstable and gives birth to the period- $2 T$ orbit. The newly born period-2T orbit is stable up to $g=65.3968$. The bifurcations to the period- $2^{2} T$, period $-2^{3} T$, period $-2^{4} T$, period- $2^{5} T$, period- $2^{6} T$, and period $-2^{7} T$ orbits are observed at $g=69.1001, g=71.7460, g=72.7732$, $g=73.0867, g=73.1587$, and $g=73.1747$. Using the above period-doubling values of $g$, we obtain Feigenbaum's ratios $\delta_{1}=1.39964, \delta_{2}=2.57596$, $\delta_{3}=3.27546, \delta_{4}=4.34436, \delta_{5}=4.55731$, and $\delta_{6}=4.66233$. The calculated $\delta$ values can be shown to approach a constant value $\delta \approx 4.66992 \ldots$, when $n \rightarrow \infty$. As $g$ increases further, a chaotic orbit and the reverse period-doubling bifurcation are found to occur. The physical reason leading to the reverse period-doubling bifurcation is the softness and reversibility of the bifurcation. The critical values of the reverse period- $2 T, 2^{2} T, 2^{3} T, 2^{4} T, 2^{5} T, 2^{6} T$, and $2^{7} T$ orbits occur at $g=110.86207, g=103.12387$, $g=101.36797, g=100.98365, g=100.90127$, $g=100.88353$, and $g=100.87872$, respectively. At $g=110.86207$, the reverse period doubling bifurcation suddenly disappears, and a long time motion settles to a periodic behavior. From the above critical values of reverse periodic orbits, we find Feigenbaum's ratios such as $\delta_{1}=4.40572, \delta_{2}=4.56885$, $\delta_{3}=4.62601, \delta_{4}=4.64375, \delta_{5}=4.65427$, and $\delta_{6}=4.66177$. In the limit $n \rightarrow \infty$, the value of $\delta$ approaches a constant value $4.66992 \ldots$. Here again, the coexistence of several attractors, the period-doubling bifurcation leading to chaotic motion, and reverse period doubling bifurcation are also found in the interval $0<g<120$.

Next, we show the effect of the control parameter $g$, by fixing the values of $f$ in a periodic region. For $f=0.1$ and $g=0$, the motion of the system is periodic with period- $T$ orbit. Figure $4, a$ is the bifurcation diagram obtained by varying $g$ in the range 42 to 54 . In Fig. 4, $a$, a transcritical bifurcation occurs at $g=43.4555$. This bifurcation persists up to $g=48.6075$. Then a period-2T solution is developed. This is followed by the bifurcations to $2^{2} T, 2^{3} T, 2^{4} T, 2^{5} T, 2^{6} T$, and $2^{7} T$ solutions and so on. The critical values of the period- $2^{2} T, 2^{3} T, 2^{4} T$, $2^{5} T, 2^{6} T$, and $2^{7} T$ orbits occur at $g=50.4256$, $g=51.0809, g=51.2648, g=51.3105, g=51.3208$, and $g=51.3231$. The onset of chaos takes place at $g=g_{c}=51.3254$. Using the values of $g$ given above, 
we obtain Feigenbaum's ratios $\delta_{1}=2.77445, \delta_{2}=$ $3.56335, \delta_{3}=4.43621, \delta_{4}==4.45578, \delta_{5}=4.46204$, and $\delta_{6}=4.46613$. These ratios approach a constant value $\delta=4.66992 \ldots$, when $n \rightarrow \infty$. When the control parameter $g$ is further increased from $g_{c}$, we could find that the chaotic motion and periodic windows persisted up to $g=54.175$. At $g=54.175$, the chaotic motion disappears. Again the period- $T$ orbit is developed in the interval $64.175<g<99.875$ (Fig. 6, a). As $g$ increases further from 99.875, the chaotic motion and reverse period-doubling bifurcation occur. At $g=175.175$, the reverse perioddoubling bifurcation disappears, and the long time motion settles to a periodic orbit (Fig. 6, a). The bifurcation of an equilibrium point into two equilibrium points at the critical value of a control parameter with one being of saddle type and the other being stable node is observed. This type of bifurcation is called the saddle-node bifurcation. In Fig. 4, we can see period-doubling windows in the bifurcation diagrams. These types of periodic orbits are developed due to the saddle-node bifurcation. In Figs. 4, $a$ and 6 , $a$, we observe the coexistence of multiple attractors, suppression, and enhancement of chaos in the interval $0<g<200$ for $f=0.1$. The bifurcation diagram corresponding to $g=75$ (chaotic motion when $f=0$ and periodic motion when $f=0.1)$ and $f \in[0,1]$ is shown in Fig. $4, b$. When the control parameter $f$ is smoothly varied, the system described by Eq. (3) starts with the chaotic motion followed by the reverse period-doubling bifurcation. As $f$ increases further, the chaotic motion and periodic window region occur in the interval $0<f<1.0$. Here again, we observe the suppression and enhancement of chaos and the existence of several attractors in the interval $0<f<1.0$ for $g=75$.

\subsection{Hysteresis}

First, we consider the effect of the high-frequency component of the force alone. In other words, $f=0$ (but $\omega \neq 0$ ), and we fix $\omega=1.0, \Omega=10.0, \beta=2.0$ and $d=0.5$. Hysteresis is observed in the presence of the high-frequency component force. The bifurcation diagram plotted by varying $g$ in the forward direction, as well as in the reverse direction, is shown in Fig. 5. Figures 5, $a$ and 5, $b$ are obtained by varying the amplitude $g$ from zero in the forward direction and from the value 200 in the reverse direc-
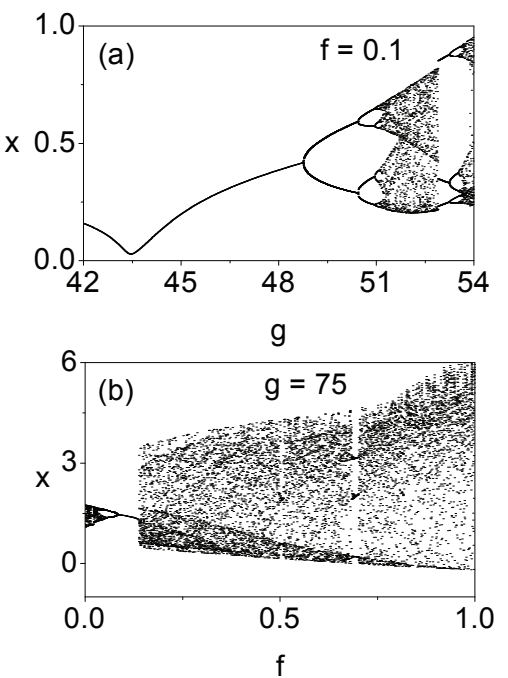

Fig. 4. Bifurcation diagrams for the system driven by an AM signal, when (a) $f=0.1$ and (b) $g=75$. The other parameters are $d=0.5, \alpha=1.0, \beta=2.0, \omega=1.0$, and $\Omega=10$

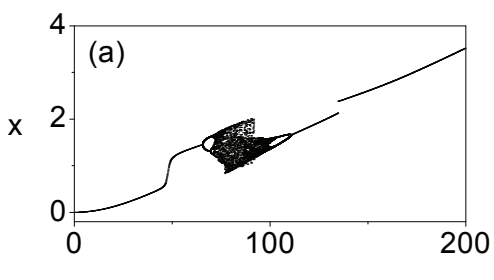

g

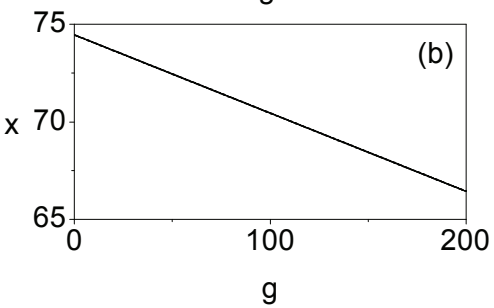

Fig. 5. Bifurcation diagrams for the system driven by an AM signal, when $g$ is varied in the $(a)$ forward direction and $(b)$ reverse direction. The other parameters are $f=0.0, d=0.5$, $\alpha=1.0, \beta=2.0, \omega=1.0$, and $\Omega=10$

tion. Different paths are followed in Figs. 5, $a$ and $5, b$. Hence, the system exhibits the hysteresis, when the control parameter $g$ is varied smoothly from a small value to a larger one and then back to the smaller value. Next, we consider the effect of the lowfrequency component of an AM signal alone, i.e., $g=0$, and the system is driven by $f \sin \omega t$. The hysteresis phenomenon is not observed in the presence of the low-frequency component of the force. 

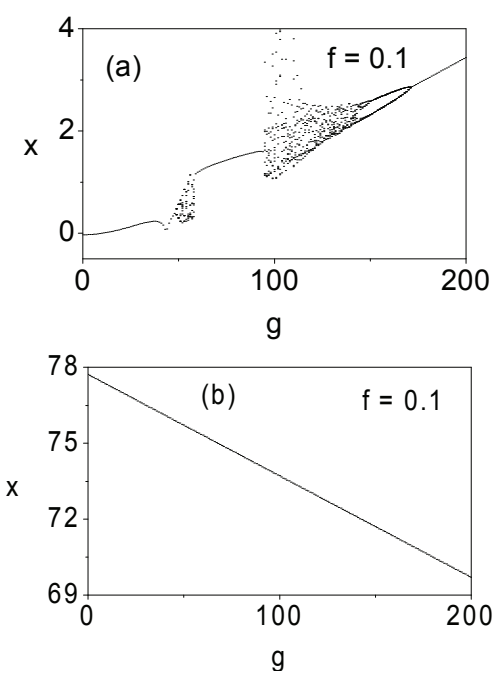

Fig. 6. Bifurcation diagrams for the system driven by an AM signal, when $g$ is varied in the forward direction $(a)$ and reverse direction $(b)$. The other parameters are $f=0.1, d=0.5, \alpha=$ $=1.0, \beta=2.0, \omega=1.0$, and $\Omega=10$
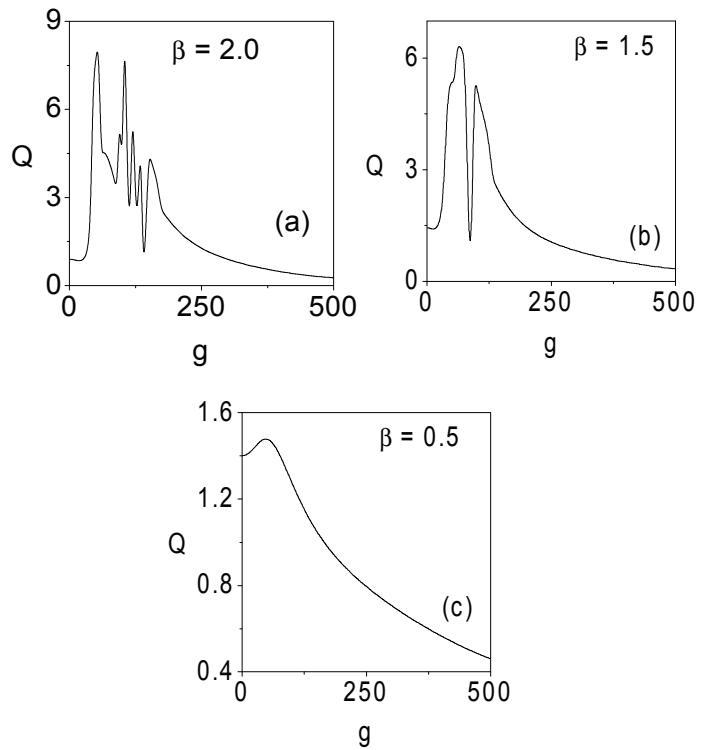

Fig. 7. Response amplitude $Q$ versus $g$ for three values of $\beta$, namely, $\beta=2.0,1.5,0.5$. The other parameters are $f=0.1$, $d=0.5, \alpha=1.0, \omega=1.0$, and $\Omega=10$

Finally, we consider the effect of both low- and high-frequency components of an AM signal, i.e., $f \neq 0$ and $g \neq 0$. We fix the parameters as $f=0.1$, $\omega=1.0, \Omega=10.0, \beta=2.0$, and $d=0.5$. Hysteresis is realized, when $g$ is varied in the forward and reverse directions in the interval $g \in[0,200]$, which is shown
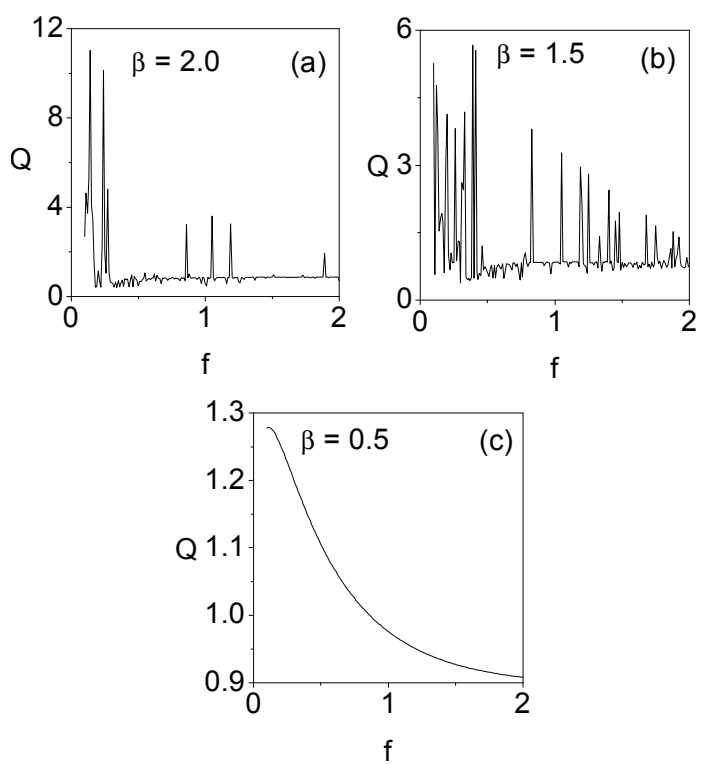

Fig. 8. Response amplitude $Q$ versus $f$ for three values of $\beta$, namely, $\beta=2.0,1.5,0.5$. The other parameters are $g=100$, $d=0.5, \alpha=1.0, \omega=1.0$, and $\Omega=10$
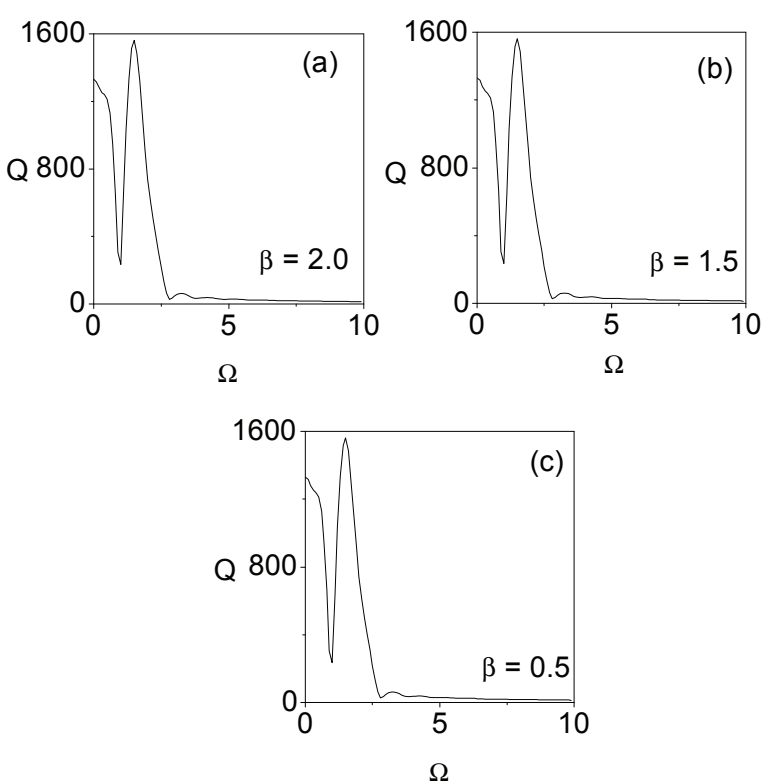

Fig. 9. Response amplitude $Q$ versus $\Omega$ for three values of $\beta$, namely, $\beta=2.0,1.5,0.5$. The other parameters are $f=0.1$, $g=100, d=0.5, \alpha=1.0$, and $\omega=1.0$

in Fig. 6. Here, we again observe the suppression and enhancement of chaos. As shown above, the presence of coexistence of regular and chaotic attractors and the hysteresis phenomenon allows us to change the behavior of the system from chaos to a regular mode

ISSN 2071-0194. Ukr. J. Phys. 2017. Vol. 62, No. 1 
by increasing the value of $g$ from the small value to a larger value and then decreasing it from the large value to a small value. The occurrence of hysteresis can be treated as a method of migration control.

\subsection{Vibrational Resonance}

In addition to the coexistence of multiple attractors and hysteresis, the system described by Eq. (3) also exhibits the phenomenon of VR, when $g$ is varied. To quantify the occurrence of $\mathrm{VR}$, we use the response amplitude $Q$ of the system at the signal frequency $\omega$. Equation (3) can be numerically integrated with the use of the fourth-order Runge-Kutta method with the time step $T=(2 \pi / \omega) / 1000$. The first $10^{3}$ drive cycles are left as transient and the values of $x(t)$ corresponding to the next 500 drive cycles are used to compute the response amplitude $(Q)$. From the numerical solution of $x(t)$, the response amplitude is computed through $Q=\sqrt{Q_{\mathrm{S}}^{2}+Q_{\mathrm{C}}^{2}} / f$, where

$$
\begin{aligned}
Q_{\mathrm{S}} & =\frac{2}{n T} \int_{0}^{n T} x(t) \sin \omega t \mathrm{~d} t, \\
Q_{\mathrm{C}} & =\frac{2}{n T} \int_{0}^{n T} x(t) \cos \omega t \mathrm{~d} t,
\end{aligned}
$$

where $T=(2 \pi / \omega)$ is the period of the response and $n$ is taken as 500 .

Figure 7 shows the variation of numerically computed $Q$ against the control parameter $g$ for three fixed values of $\beta$, namely, $\beta=2.0,1.5,0.5$. The values of other parameters are $\alpha=1, d=0.5, f=0.1, \omega=1$ and $\Omega=10$. The $Q$ value increases with $g$, reaches a maximum, and then decreases, as $g$ further increases. The underlying phenomenon is VR, since the occurrence is due to the high-frequency component of the force. In Fig. 7, the number of resonances decreases with decrease in $\beta$. Five resonances occur for $\beta=2.0$, which is clearly shown in Fig. 7, $a$. The first resonance occurs at $g=51.322$ with $Q_{\max }=8.3216$, second resonance at $g=105.555, Q_{\max }=7.6$, third resonance at $g=122.751, Q_{\max }=5.2195$, fourth resonance at $g=134.656, Q_{\max }=4.07619$, and fifth resonance at $g=153.1741$ with $Q_{\max }=4.2666$. When $g>175$, no resonance occurs. In Fig. $7, b$, for $\beta=$ $=1.5$, only two resonances occur. The first one occurs at $g=66.13756$ with $Q_{\max }=6.28148$, second at $g=99.2061$ with $Q_{\max }=5.2449$, and no resonance occurs, when $g>125$. For $\beta=0.5$, only one resonance occurs at $g=46.82539$ with $Q_{\max }=1.476825$, which is presented in Fig. 7, $c$, and no resonance occurs, when $g>62.5$.

We analyze the influence of the parameters $f$ and $\Omega$ on the resonance. The results are presented in Figs. 8 and 9 . $Q$ versus $f$ is plotted in Fig. 8 for different values of $\beta$, namely, $\beta=2.0,1.5,0.5$ and with $g=100$, $\omega=1$ and $\Omega=10$. Multiple resonances occur for $\beta=2.0$ and 1.5 (Figs. 8, $a$ and $b$ ), and no resonance occurs for $\beta=0.5$ (Fig. 8, c). In Fig. 8, $c, Q$ is found to decrease with increase in $f$. The dependence of $Q$ on the frequency $\Omega$ of the driving force is presented in Fig. 9 for different values of $\beta$, namely $\beta=2.0,1.5$, 0.5 and with $\omega=1, f=0.1$ and $g=100$. In all the cases, only two resonances occur with almost equal $Q_{\max }$ value.

\section{Conclusion}

We have numerically studied the dynamics of the classical Morse oscillator driven by an AM signal for a specific set of values of the parameters. The coexistence of several attractors, bifurcations of them, and the phenomena of hysteresis and vibrational resonance are found. Various types of bifurcations such as transcritical, reverse period-doubling, etc. are encountered in the system (Eq. 3). Small windows of chaotic behavior separated by regions of periodic mode are found. The occurrence of VR depends on the control parameters $\beta, f, g$, and $\Omega$. From our numerical analysis, we observe multiple resonant peaks for different values of the control parameters such as $f, g$, and $\Omega$. The amplitude modulated force considered in the present work has four parameters such as $\omega, \Omega, f$, and $g$. As shown in Figs. 2, 4, and 6, the presence of additional parameters can be used to suppress or enhance chaos.

It is of interest to investigate certain nonlinear phenomena such as the hysteresis, chaos, vibrational resonance, ghost vibrational resonance, etc. in the system driven by narrow- and wide-band frequencymodulated signals. The account for noise induced in the system may lead to some interesting results. The work along this direction is in progress.

We thank two anonymous referees, whose constructive criticisms have led to qualitative improvements in the manuscript. 
1. P.M. Morse. Diatomic molecules according to the wave mechanics. II. Vibrational levels. Phys. Rev. E 34, 57 (1929) [DOI: 10.1103/PhysRev.34.57].

2. G. Herzberg. Spectra of Diatomic Molecules (Van Nostrand, 1950).

3. M.E. Goggin, P.W. Milonni. Driven Morse oscillator: Classical chaos, quantum theory, and photodissociation. Phys. Rev. A 37, 796 (1988) [DOI: 10.1103/PhysRevA.37.796].

4. J.R. Ackerhalft, P.W. Milonni. Chaos and incoherence in a model of multiple-photon excitation of molecular vibrations. Phys. Rev. A 34, 1211 (1986) [DOI: 10.1103/PhysRevA.34.1211].

5. P.S. Dardi, S.K. Gray. Classical and quantum mechanical studies of hydrogen fluoride in an intense laser field. J. Chem. Phys. 77 (3), 1345 (1982) [DOI: 10.1063/1.443957].

6. S.K. Gray. Classical aspects of laser excitation of a Morse oscillator. J. Chem. Phys. 75 (1), 67 (1983) [DOI: 10.1016/0301-0104(83)85008-3].

7. K.M. Christoffel, J.M. Bowman. Classical trajectory studies of multiphoton and overtone absorption of hydrogen fluoride. J. Phys. Chem. 85 (15), 2159 (1981) [DOI: 10.1021/j150615a004].

8. D. Beigie, S. Wiggins. Dynamics associated with a quasiperiodically forced Morse oscillator: Application to molecular dissociation. Phys. Rev. A 45 (7), 4803 (1992) [DOI: 10.1103/PhysRevA.45.4803].

9. K. Abirami, S. Rajasekar, M.A.F. Sanjuan. Vibrational resonance in the Morse oscillator. Pramana J. of Phys. 81 (1), 127 (2013) [DOI: 10.1007/s12043-013-0546-z].

10. S. Behnia, A. Akhshani, M. Panahi, R. Asadi. Controlling chaos in damped and driven Morse oscillator via slavemaster feedback. Acta Physica Polon. A 123, 7 (2013) [DOI: 10.12693/APhysPolA.123.7].

11. Z. Jing, J. Deng, J. Yang. Bifurcations of periodic orbits and chaos in damped and driven Morse oscillator. Chaos, Solitons and Fractals 35, 486 (2008) [DOI: 10.1016/j.chaos.2006.05.038].

12. T. Kapitaniak, Yu. Maistrenko. Multiple choice bifurcations as a source of unpredictability in dynamical systems. Phys. Rev. E 58 (4), 5161 (1998) [DOI: 10.1103/PhysRevE.58.5161].

13. A. Ray, D. Ghosh, A.R. Chowdhury. Topological study of multiple coexisting attractors in a nonlinear system. J. Phys. A: Math. Theor. 42 (38), 385102 (2009) [DOI: 10.1088/1751-8113/42/38/385102].

14. M. Dutta, H.F. Nusse, E. Ott, J.A. Yorke, G.H. Yuan. Multiple attractor bifurcations: A source of unpredictability in piecewise smooth systems. Phys. Rev. Lett. 83 (21), 4281 (1999) [DOI: 10.1103/PhysRevLett.83.4281].

15. K. Sun, A. Di-li Duo Li-Kun, Y. Dong, H. Wang, K. Zhong. Multiple coexisting attractors and hysteresis in the generalized Ueda oscillator. Mathematical Problems in Engineering, Article ID 256092 (2013) [DOI: 10.1155/2013/256092].
16. V.M. Gandhimathi, S. Rajasekar, J. Kurths. Vibrational and stochastic resonances in two coupled overdamped anharmonic oscillators. Phys. Letts. A 360, 279 (2006) [DOI: 10.1016/j.physleta.2006.08.051].

17. K. Abirami, S. Rajasekar, M.A.F. Sanjuan. Vibrational and ghost-vibrational resonances in a modified Chua's circuit model equation. Int. J. Bifurcation Chaos 24, 1430031 (2014) [DOI: 10.1142/S0218127414300316].

18. O. de Feo, G. Mario Maggio. Bifurcations in the colpitts oscillator: From theory to practice. Int. J. Bifurc. Chaos 13, 2917 (2003) [DOI: 10.1142/S0218127403008338].

19. V.P. Lukomsky, I.S. Gandzha. Cascades of subharmonic stationary states in strongly non-linear driven planar systems. J. Sound and Vibration 275 (1), 351 (2004) [DOI: 10.1016/j.jsv.2003.06.029].

20. L. Gammaitoni, P. Hanggi, P. Jung, F. Marchesoni. Stochastic resonance. Rev. Mod. Phys. 70, 223 (1998) [DOI: 10.1103/RevModPhys.70.223].

21. P.S. Landa, P.V.E. McClintock. Vibrational resonance. J. Phys. A: Math. Gen. 33, L433 (2000) [DOI: 10.1088/ 0305-4470/33/45/103].

22. S. Jeyakumari, V. Chinnathambi, S. Rajasekar, M.A.F. Sanjuan. Single and multiple vibrational resonance in a quintic oscillator with monostable potentials. Phys. Rev. E 80, 046608 (2009) [DOI: 10.1103/PhysRevE.80.046608].

23. S. Rajasekar, K. Abirami, M.A.F. Sanjuan. Novel vibrational resonance in multistable systems. Chaos 21, 033106 (2011) [DOI: 10.1063/1.3610213].

24. E. Ullner, A. Zaikin, J. García-Ojalvo, R. Báscones, J. Kurths. Vibrational resonance and vibrational propagation in excitable systems. Phys. Lett. A 312, 348 (2003) [DOI: 10.1016/S0375-9601(03)00681-9].

25. A.A. Zaikin, L. Lopez, J.P. Baltanas, J. Kurths, M.A.F. Sanjuan. Phys. Rev. E 66, 011106 (2002).

26. S. Rajasekar, J. Used, A. Wagemakers, M.A.F. Sanjuan. Vibrational resonance in biological nonlinear maps. Commun. Nonlinear Sci. Numer. Simulat. 17, 3435 (2012) [DOI: 10.1016/j.cnsns.2011.12.014].

27. C. Jeevarathinam, S. Rajasekar, M.A.F. Sanjuan. Theory and numerics of vibrational resonance in Duffing oscillators with time-delayed feedback. Phys. Rev. E 83, 066205 (2011) [DOI: 10.1103/PhysRevE.83.066205].

28. J.H. Yang, M.A.F. Sanjuan, H.G. Liu. Vibrational subharmonic and superharmonic resonances. Commun. Nonlinear Sci. Numer. Simulat. 30, 362 (2016) [DOI: 10.1016/ j.cnsns.2015.07.002].

29. V. Ravichandran, V. Chinnathambi, S. Rajasekar. Homoclinic bifurcation and chaos in Duffing oscillator driven by an amplitude-modulated force. Physica A 376, 223 (2007) [DOI: 10.1016/j.physa.2006.11.003].

30. J.H. Yang, X.B. Liu. Controlling vibrational resonance in a delayed multistable system driven by an amplitude-

ISSN 2071-0194. Ukr. J. Phys. 2017. Vol. 62, No. 1 
modulated signal. Phys. Scr. 82, 025006 (2010) [DOI: 10.1088/0031-8949/82/02/025006].

31. V.M. Gandhimathi, S. Rajasekar. Vibrational and stochastic resonances in two coupled overdamped anharmonic oscillators driven by an amplitude modulated force. Phys. Scr. 76, 693 (2007) [DOI: 10.1088/0031-8949/76/ 6/019].

32. M.J. Feigenbaum. Quantitative universality for a class of nonlinear transformations. J. Stat. Phys. 19, 25 (1978) [DOI: 10.1007/BF01020332].

33. M.J. Feigenbaum. Universal behavior in nonlinear systems. Los Alamos Science 1, 4 (1980).

Received 26.12.15
С. Гурупаран, В. Равічандран, В. Чінатамбі, С. Раджасекар СПIВIСНУВАННЯ КРАТНИХ АТТРАКТОРІВ, ГІСТЕРЕЗИСУ ТА КОЛИВАЛЬНОГО РЕЗОНАНСУ В КЛАСИЧНОМУ ОСЦИЛЯТОРІ МОРСА, ЩО ЗБУДЖУЄТЬСЯ СИГНАЛОМ, 3 МОДУЛЬОВАНОЮ АМПЛІТУДОЮ

Р е з ю м е

Розглянуто класичний осцилятор Морса, що збуджується сигналом, $з$ амплітудною модуляцією на двох суттєво різних частотах $\omega$ і $\Omega$ з $\Omega \gg \omega$. Чисельно розраховано динаміку осцилятора для конкретного набору параметрів. Показано співіснування кількох $T$-періодичних орбіт, їx біфуркації, і явища гістерезису і коливального резонансу. Представлено характеристику періодичних і хаотичних орбіт, гістерезису і коливального резонансу з використанням діаграми біфуркацій та амплітуди відгуку. 